\title{
CLUSTERS OF SMALL ERUPTIVE FLARES PRODUCED BY MAGNETIC RECONNECTION IN THE SUN
}

\author{
V. ARCHONTIS ${ }^{1}$ AND V. HANSTEEN ${ }^{2}$ \\ ${ }^{1}$ School of Mathematics and Statistics, St. Andrews University, St. Andrews, KY16 9SS, UK; vasilis@mcs.st-and.ac.uk \\ 2 Institute of Theoretical Astrophysics, University of Oslo, NO-0315 Oslo, Norway \\ Received 2014 March 12; accepted 2014 April 27; published 2014 May 20
}

\begin{abstract}
We report on the formation of small solar flares produced by patchy magnetic reconnection between interacting magnetic loops. A three-dimensional (3D) magnetohydrodynamic (MHD) numerical experiment was performed, where a uniform magnetic flux sheet was injected into a fully developed convective layer. The gradual emergence of the field into the solar atmosphere results in a network of magnetic loops, which interact dynamically forming current layers at their interfaces. The formation and ejection of plasmoids out of the current layers leads to patchy reconnection and the spontaneous formation of several small (size $\approx 1-2 \mathrm{Mm}$ ) flares. We find that these flares are short-lived ( $30 \mathrm{~s}-3$ minutes) bursts of energy in the range $O\left(10^{25}-10^{27}\right)$ erg, which is basically the nanoflare-microflare range. Their persistent formation and co-operative action and evolution leads to recurrent emission of fast EUV/X-ray jets and considerable plasma heating in the active corona.
\end{abstract}

Key words: magnetohydrodynamics (MHD) - Sun: activity - Sun: corona - Sun: flares - Sun: magnetic fields

Online-only material: animations, color figures

\section{INTRODUCTION}

Observations (e.g., Lin et al. 1984; Porter et al. 1987; Hannah et al. 2008) have revealed the existence of numerous microflares (transient brightenings with energy $O\left(10^{27}\right)$ erg and size smaller than the standard flares (e.g., Priest \& Forbes 2002; Shibata \& Magara 2011) on the Sun. The areas around microflares are often bright in X-rays, which implies plasma heating (e.g., Porter et al. 1987; Hudson 1991). Thus, microflares have been considered as possible sources for heating the solar corona (e.g., Lin et al. 1984; Porter et al. 1987), subject to their occurrence rate and energy release. On theoretical grounds, Parker (1988) suggested that the active X-ray corona consists of numerous nanoflares $\left(O\left(10^{24}\right) \mathrm{erg}\right.$, with the largest nanoflares approaching $10^{26}-10^{27} \mathrm{erg}$ ) and that microflares could be made up of several nanoflares. Also, previous two-dimensional (2D) simulations (Yokoyama \& Shibata 1995) and observations (Chae et al. 1999) have shown that X-ray jets and cooler $\mathrm{H}_{\alpha}$ surges can be emitted from microflares, possibly due to reconnection between emerging and pre-existing coronal magnetic fields. All the above suggest that although nano/microflares are small-scale events, they have a great influence on the solar atmosphere. However, their three-dimensional (3D) formation, evolution, and energetics are not well known. Moreover, the existing standard flare models (e.g., Sweet 1969; Kopp \& Pneuman 1976; Shibata et al. 1995) elaborate the onset of larger and single flares in coherent (almost monolithic) current sheets and, therefore, cannot address simultaneously the onset of nano/ microflares and the intermittent heating (e.g., Machado et al. 1988; Parker 1988; Isobe et al. 2005; Nishizuka et al. 2010) of the solar corona.

Here we report radiative magnetohydrodynamic (MHD) simulations, showing that small flares are formed naturally by patchy reconnection, which is triggered by the eruption of plasmoids (e.g., Tsuneta 1997; Shibata \& Tanuma 2001; Archontis et al. 2006) in fragmented current sheets, between interacting magnetic bipoles (e.g., Machado et al. 1988; Hanaoka 1996). The fragmentation of currents explains naturally the ubiquitous intermittent heating and filamentary nature (e.g., Howard \& Stenflo 1972; Isobe et al. 2005) of the solar corona. Moreover, we find that the frequent onset and co-operative action of small flares dump enough energy in the solar atmosphere, sufficient to accelerate and heat the plasma in the active corona.

\section{THE MODEL}

We solve the 3D time-dependent, resistive MHD equations in Cartesian geometry, using the Bifrost code (Gudiksen et al. 2011). External resistivity is computed using a hyper-diffusion operator (Gudiksen et al. 2011), which focuses dissipation in regions with steep field gradients. Our experiment is performed in a model that includes the upper convection zone (with a depth of $z=-2.5 \mathrm{Mm}$ below the photosphere), the photosphere/ chromosphere $\left(z=0-2.5 \mathrm{Mm}\right.$ and temperature $T=5 \times 10^{3}-$ $O\left(10^{5} \mathrm{~K}\right)$, the transition region where the temperature increases rapidly with height, and the $\left(O\left(10^{6}\right) \mathrm{K}\right)$ corona that starts at $z \approx 4 \mathrm{Mm}$ above the photosphere).

The numerical domain is $24 \times 24 \times 17 \mathrm{Mm}$ in the transverse $(x)$, longitudinal $(y)$, and vertical $(z)$ directions, respectively, and it is resolved by $504 \times 504 \times 496$ grid points. In height, the resolution is $\approx 20 \mathrm{~km}$ from the photosphere to the lower corona increasing to $\approx 100 \mathrm{~km}$ in the upper corona and lower convection zone. In $x$ and $y$, the resolution is uniform $(\approx 48 \mathrm{~km})$. In the present experiments, convection is driven by optically thick radiative transfer from the photosphere. Radiative losses in the lower chromosphere include scattering and are assumed optically thin at greater heights (Carlsson \& Leenaarts 2012). Field-aligned thermal conduction is included. The model is initialized with a uniform oblique magnetic field $(B<0.1 \mathrm{G})$ that fills the corona, making an inclination angle of $45^{\circ}$ with respect to the $z$-axis. First, the experiment runs until a relatively steady state equilibrium is achieved. Then, to model flux emergence, a uniform magnetic flux sheet with $B_{y}=3360 \mathrm{G}$ is inserted into the lower boundary, within the domain $[x, y]=$ [0-24, 3-16] Mm, for a time period of 105 minutes.

\section{RESULTS AND DISCUSSION}

The embedded magnetic flux sheet is distorted by convective upflows/downflows developing loops, which eventually emerge to the photosphere as magnetic bipoles with mixed polarity. 


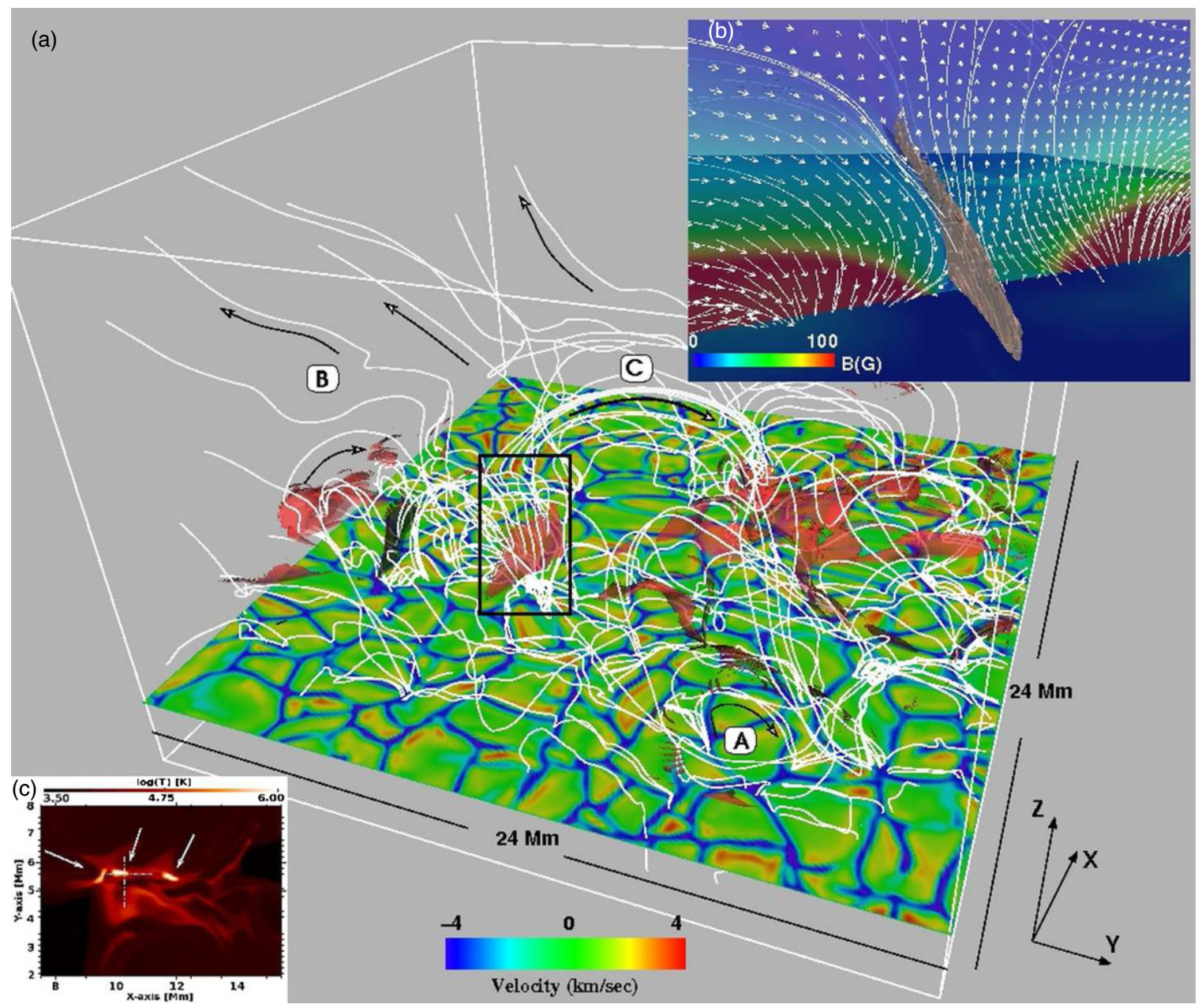

Figure 1. (a) Field lines show the magnetic topology of the emerging field at $t=7800 \mathrm{~s}$. Current sheets (transparent isosurfaces with current density $\left(0.042 \mathrm{~A} \mathrm{~m}^{-2}\right)$ are formed between interacting loops. Highly bent field lines (e.g., B) is the result of reconnection between emerging and pre-existing magnetic fields. The arrows (black) show the direction of the magnetic field along the field lines. The horizontal slice $(z=0.28 \mathrm{Mm})$ shows the photospheric flows as a result of granular convection. (b) Close-up of the interface current, which is outlined by the rectangular inset in panel (a). The field lines of the neighboring magnetic loops start to reconnect at the current sheet. The full magnetic field vector (arrows in the range $3.4 \leqslant y \leqslant 7.3 \mathrm{Mm}, 0.62 \leqslant z \leqslant 4.28 \mathrm{Mm}, x=10.35 \mathrm{Mm}$ ) shows the oppositely directed and highly sheared field across the current. (c) Temperature distribution at $z=4.32 \mathrm{Mm}(t=8770 \mathrm{~s})$ reveals a cluster of three small flares that form along the interface shown in panel (b). Dashed lines (at $x=10.35 \mathrm{Mm}$ and $y=5.6 \mathrm{Mm}$ ) show the position of the 2D slices in Figure 2.

(A color version of this figure is available in the online journal.)

The emergence of the magnetic field above the photosphere is due to the onset of the magnetic buoyancy instability, as has been shown in previous experiments of magnetic flux emergence (e.g., Archontis et al. 2004; Sykora et al. 2008). Figure 1(a) shows that the emergence and the expansion of the bipoles above the photosphere produces a multi-scale network of magnetic loops (e.g., A $(\approx 1-2 \mathrm{Mm})$ and $\mathrm{C}(\approx 7-8 \mathrm{Mm})$ ). Reconnection between the emerging loops and the oblique preexisting field opens the way for the plasma ejecta, which occur during the evolution of the system, to move toward the outer solar atmosphere.

Several of the magnetic loops are interconnected via the same field lines in a "sea-serpent" manner. Profound interaction occurs between magnetic bipoles that expand and press against each other (Figure 1(b)). Their oppositely directed field lines come closer together and strong current layers are built up at their interface. The field across the interface is strongly sheared. The resistivity is locally enhanced in the current layers and efficient reconnection of the sheared field leads to dissipation of energy and triggering of small flares (Figure 1(c)).

The onset of a flaring event is illustrated in Figure 2, which shows the plasma evolution across the interface current (visualized in Figure 1(b)). For $t \geqslant 8530 \mathrm{~s}$, the interacting magnetic loops have expanded into the corona. Figure 2(a) shows the cool adiabatic expansion of the two interacting loops (e.g., $y \approx 2 \mathrm{Mm}, z \approx 4 \mathrm{Mm}$ and $y \approx 10 \mathrm{Mm}, z \approx$ $4 \mathrm{Mm})$. At the interface, the current develops into a very long and thin layer (Figure 2(c)), which becomes subject to the resistive tearing instability leading to the formation of cool and dense (Figures 2(a) and (b)) magnetic "islands" (i.e., plasmoids; e.g., Tsuneta 1997; Shibata \& Tanuma 2001; Archontis et al. 2006) with X-type reconnection points in between. Eventually (Figures 2(e) and (h)), the plasmoids move out of the current layer and flux from the emerging loops comes into the interface and reconnects. This facilitates the eruption of plasmoids, the emission of successive bi-directional flows (jets; Figures 2(f) and (i)), and the disclosure of a smallscale but intense brightening (i.e., flaring event; Figure 2(g)) where the plasma is heated to X-ray temperatures due to Joule dissipation via reconnection. After the eruption of the plasmoids we measure the ratio of the inflow toward the diffusion region to the Alfvén speed around the reconnection site (see, e.g., Archontis et al. 2007 for a similar study), which is an indication of the reconnection rate. The average value of this ratio during 

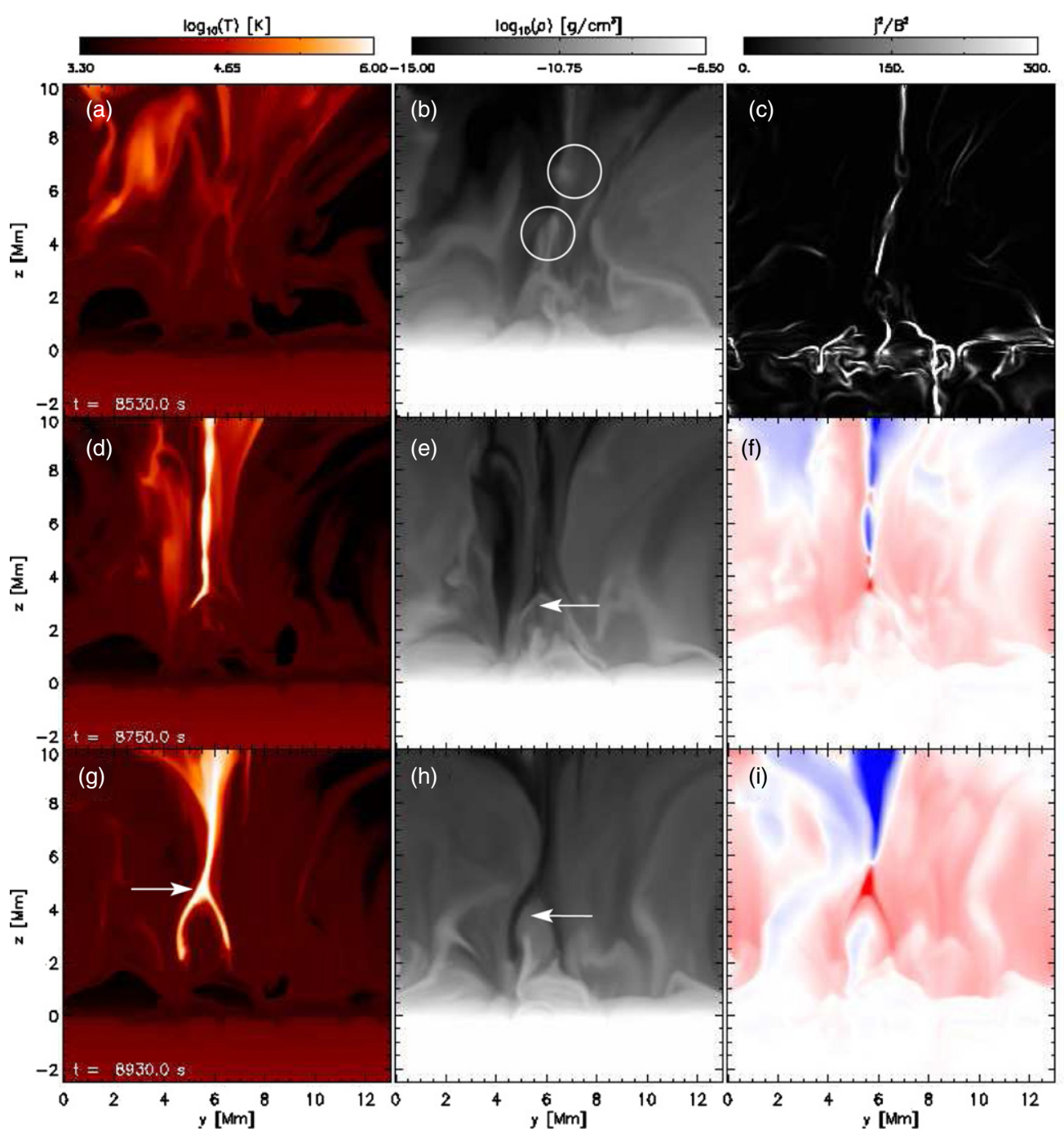

Figure 2. Evolution across the interface at $x=10.35 \mathrm{Mm}$. Times are $t=8530 \mathrm{~s}, t=8750 \mathrm{~s}$, and $t=8930 \mathrm{~s}$ (top to bottom). Panels (f) and (i) show $v_{z}$ in the range $[-100,100] \mathrm{km} \mathrm{s}^{-1}$ (red/blue). Two plasmoids are outlined in panel (b). Arrows (panels (e) and (h)) show the post-flare loop and the approximate site of the flare $(z \approx 4.7 \mathrm{Mm}$, panel $(\mathrm{g}))$.

(An animation and a color version of this figure are available online.)

the dynamical evolution of the interface is $\approx 0.1$, which indicates that the reconnection is fast. Typical values of plasma $\beta$, around the reconnection regime at the interface, lie in the range $0.01-0.1$.

The upward product of reconnection is a jet with a velocity of $\approx 200 \mathrm{~km} \mathrm{~s}^{-1}$ (Figure 2(i)) and temperature up to $\approx 2.5 \mathrm{MK}$. This emission can be observed as a (soft) X-ray jet. The reconnected field lines that are released downward form a small post-flare loop (Figures 2(e) and (h)), which is heated at the top $\left(\approx 2 \times 10^{6} \mathrm{~K}\right)$ by the collision of the downward reconnection jet with the local plasma. We find that reconnection generates slow-mode shocks that are attached to the ends of the diffusion region (e.g., at $z \approx 5.5 \mathrm{Mm}$; Figure 2(i)). They exist close (and along) to the edges of the two downflows (shown in Figure 2(i)), which adopt an overall inverse $\mathrm{V}$-shape configuration. The slow shocks is a characteristic feature of the Petschek-type reconnection. Most of the conversion of the magnetic energy into heat and bulk kinetic energy (via reconnection) occurs at these shocks. We also find a fast termination shock, which is the result of the collision between the downward reconnection outflow and the post-reconnection loops. In
Figure 2(i), the termination shock is found at $z \approx 4.5 \mathrm{Mm}$. Slow and fast shocks are formed repeatedly during the dynamical evolution of the interface. The energy released by reconnection is transported along the reconnected field lines via thermal conduction, causing flaring of the loop (Figure 2(g)) and heating of the transition region/chromospheric plasma at its footpoints. As magnetic reconnection proceeds in the corona, newly reconnected field lines successively pile up on the post-flare loop, which consequently adopts a cusp-like shape (Figures $2(\mathrm{~h})$ and (i); see also Figure 3(d)). The lifetime of the event (from flaring to cooling by radiation and conduction) is $\leqslant 100 \mathrm{~s}$ and the corresponding energy release is $10^{25}-10^{26} \mathrm{erg}$.

A similar process to the above has been invoked by observations (Masuda et al. 1994; Tsuneta 1997) and theoretical models (e.g., Sweet 1969; Kopp \& Pneuman 1976; Shibata et al. 1995) to explain large (length $\approx 100 \mathrm{Mm}$ ) standard (e.g., two-ribbon) flares associated with the eruption of filaments or large plasmoids. Here, we have shown that this mechanism also occurs on much smaller scales $(\approx 1-2 \mathrm{Mm})$ and that it might account for the onset of nano/microflares. Moreover, the present model shows that not only one but a cluster of small flares occur along 

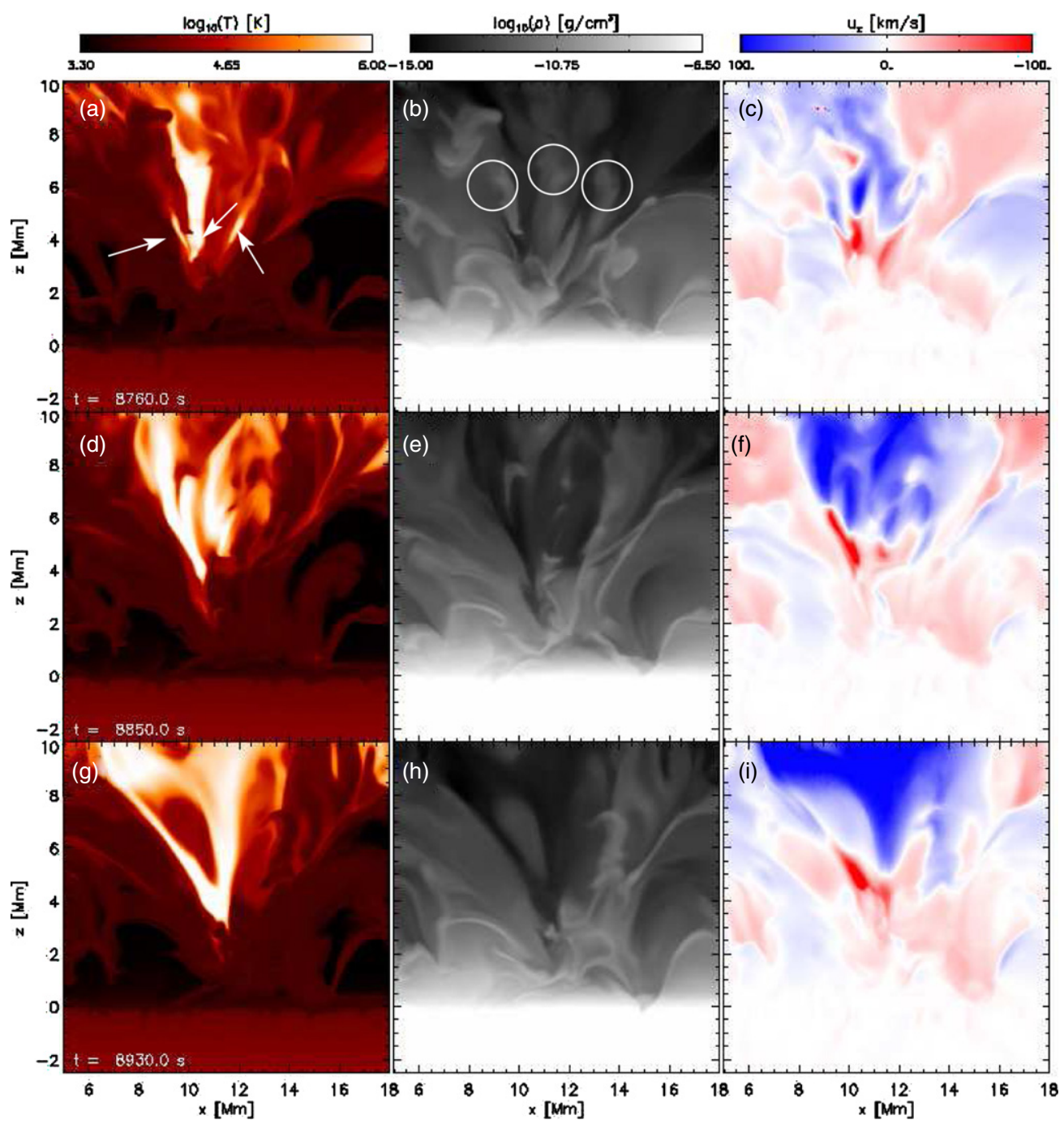

Figure 3. Evolution along the interface at $y=5.6 \mathrm{Mm}$. Times are $t=8760 \mathrm{~s}, t=8850 \mathrm{~s}$, and $t=8930 \mathrm{~s}$ (top to bottom). Segments of three plasmoids are outlined in panel (b). The same plasmoids are visualized in the 3D space in Figure 4(a). Underneath the plasmoids, there are individual small flare sites (shown by arrows in panel (a)).

(An animation and a color version of this figure are available online.)

the 3D current layer. This is illustrated in Figures 3 and 4 . At one time, several plasmoids can exist in the same interface. They are identified by, e.g., a local enhancement in density (encircled in Figure 3(b)). Their ejection leads to patchy reconnection, which in turn gives onset to spatially intermittent heating (Figures 3(c) and (f)) and initiates the individual small flares (e.g., Figure 3(a), arrows).

Due to the tearing instability and the dynamic evolution (including coalescence) of the plasmoids, the current layer undergoes fragmentation. It breaks up into hot "filaments" of intense current (shown later in Figure 4(c)), which in fact are smaller dissipation sites emitting distinct bi-directional flows (Figures 3(c) and (f)). The above results could explain naturally the intermittent heating in the active solar corona (e.g., Machado et al. 1988). On larger scales, a similar mechanism might operate to produce the observed multiple downflows above flare loops (e.g., Asai et al. 2004; McKenzie \& Savage 2009). These plasma properties cannot be explained by the traditional flare models, which rely on the existence of single ("monolithic") current sheets.

The further evolution of the system along the interface reveals an interesting result. For $t \geqslant 8850 \mathrm{~s}$, the individual bursts start to become indistinguishable from one another (Figures 3(d) and $(\mathrm{g}))$. Since the distance between them is very short, the onset and evolution of one flare affects (even stimulates) the other by reconfiguring the nearby magnetic field topology. As a result, we are witnessing a gradual blending of the small flares and the development of another (larger in energy release) flare. Therefore, the explosive flaring at $t=8930 \mathrm{~s}$ (Figure $3(\mathrm{~g})$ ) is not an all-time individual brightening but it is the composite effect of the adjacent small flares. Indeed, the onset of the flare at $t=8930 \mathrm{~s}$ is spatially and temporally associated with the joint eruption of the neighboring plasmoids. The eruption is thrusted by a V-shaped reconnection jet (Figure 3(i)).

Figure 4(a) shows the eruption of plasmoids and the intermittent heating at the interface in the $3 \mathrm{D}$ space. The (blue) isosurfaces illustrate the dense core of the plasmoids. Underneath, there are the hot filamentary structures of the fragmented current layer. Small flares are produced at the lower part of each filament (see later in Figure 4(d)). The magnetic field lines show the highly twisted nature of the field in and around the plasmoids. The individual plasmoid field lines reconnect rapidly where they intersect and, thus, they become stochastic as they pass through successive plasmoids: field lines (e.g., white and red) go through 


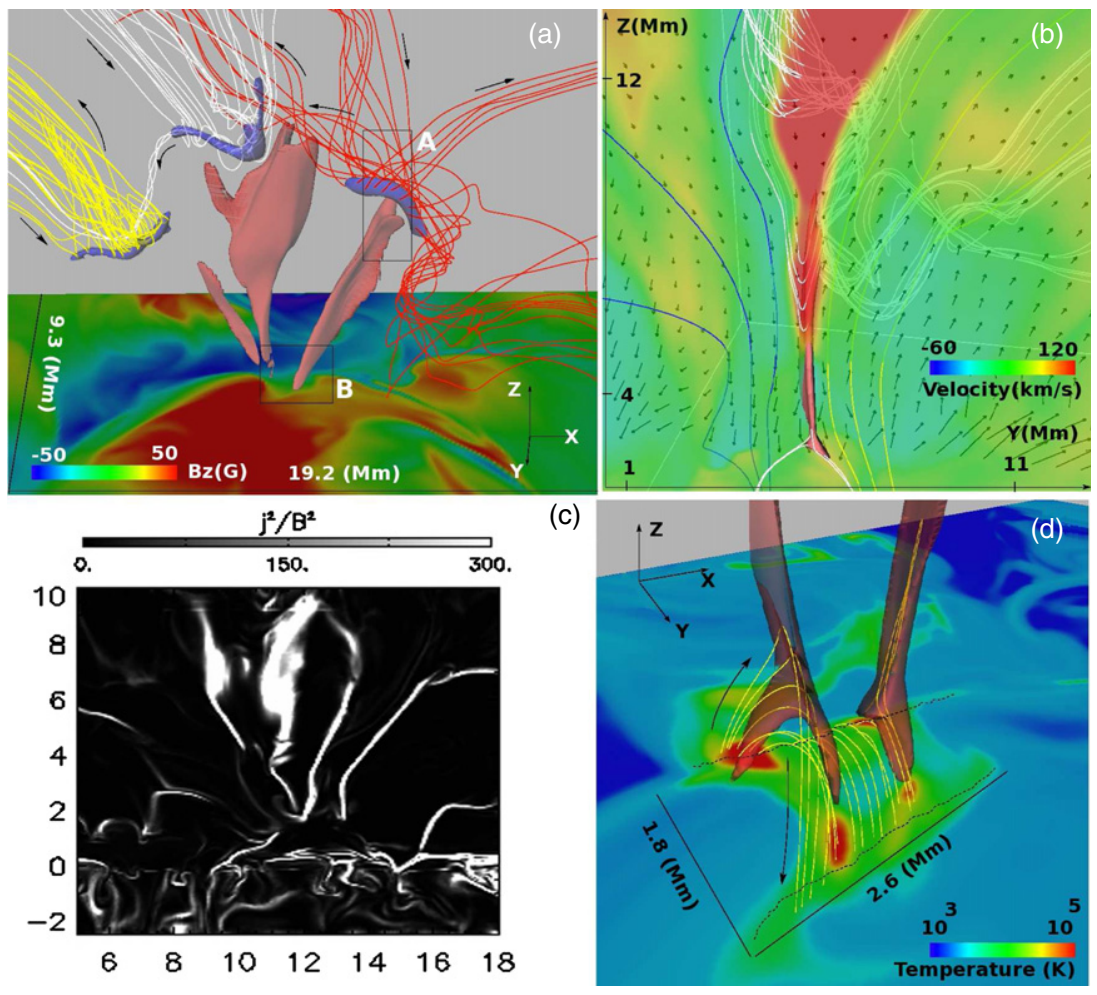

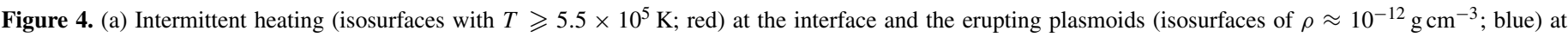

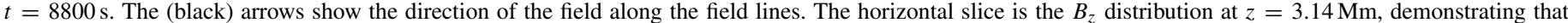

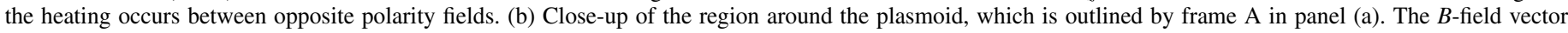

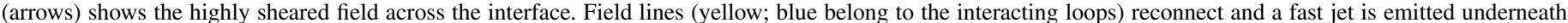

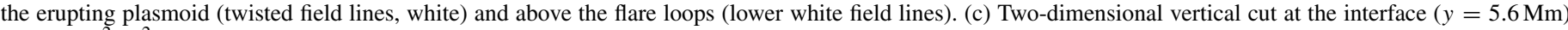

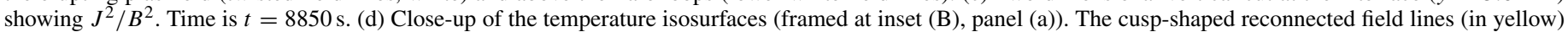
show the topology of the post-flare loops. The dashed lines indicate the length of the small two-ribbon flare.

(A color version of this figure is available in the online journal.)

the close vicinity of (at least) two plasmoids in succession. This implies that the eruption of one plasmoid can affect or even initiate the eruption of the others. Accordingly, each flare can change the field line topology so that other sites start to reconnect and another flare is set off nearby. This can lead to "sympathetic" flaring activity and, ultimately, to profound heating and plasma expulsion as we discussed earlier.

The eruption of plasmoids evolves into helical jets. This is partially illustrated in Figure 4(b). The upward fast reconnection jet (red) consists of the twisted field lines (white) of the plasmoid on its upper part (at $z \approx 12 \mathrm{Mm}$ ). During the emission, the erupted plasmoids come into contact with the "open" ambient (non-twisted) magnetic field and reconnect. This leads to the formation of helical jets and an untwisting motion along the jets (for similar studies, see, e.g., Shimojo et al. 2007; Archontis \& Hood 2013). Figure 4(b) also shows that the hot reconnection plasma underneath the plasmoid is ejected upward by the tension of the reconnected ( $\mathrm{V}$-shaped, white) field lines at the interface. During the evolution, similar reconnection events occur at various atmospheric heights (from the chromosphere up to the corona) and, thus, we detect the emission of several EUV and $\mathrm{X}$-ray jets from the interface. Many of the hot reconnection jets, which are produced at the interface, have velocities comparable to the local Alfvén speed.

Figure 4(c) displays $J^{2} / B^{2}$ at $y=5.6 \mathrm{Mm}$ (the same vertical cut as in Figure 3). It is shown that the interface current is not uniform and coherent but it is fragmented. It consists of thin, individual, filamentary structures of strong current where efficient reconnection occurs. A comparison with Figure 4(a) shows that there is a good spatial relationship between the sites of profound heating and the places with strong current. This implies that the intermittent heating is due to the fragmentation of the interface current layer.

Figure 4(d) displays the lower part of the hot filaments (inverse Y-shaped temperature isosurfaces, $T \geqslant 5 \times 10^{5} \mathrm{~K}$ ). Reconnected field lines form an arcade of loops. The overall arcade is not the result of a single eruptive flare, as has been previously thought, but it consists of distinct, cusp-like, small eruptive flares, which show up as "miniature" version of bigger flares (e.g., Masuda et al. 1994; Tsuneta 1996, 1997). Two bright ribbons form on either side of the neutral line, joining the successive footpoints of the post-flare loops. Thus, a cluster of small eruptive flares can form a tiny two-ribbon flare.

The powering of the flares at the interface occurs repetitively over a 40 minute period: we find more than 20 flares at $t \approx 8400-10,800 \mathrm{~s}$. The heat and energy input (Figures 5(a) and (b)) to the chromosphere/corona is highly intermittent, with profound fluctuations in temperature as the plasma is suddenly heated by small flares and subsequently cools down. During the above period, there is a remarkable temporal (and spatial) correlation among heating, energy release, and the onset of fast $\left(200-400 \mathrm{~km} \mathrm{~s}^{-1}\right)$ jets. This is a direct evidence of reconnection-driven plasma acceleration in small eruptive flares. Similar evolution is found in other interfaces within the numerical domain. The flares produced appear at random intervals, with an average lifetime of $\approx 30 \mathrm{~s}-3$ minutes. They occur at various atmospheric heights (chromosphere-corona) and they are capable of heating the plasma to $\approx 1-6 \mathrm{MK}$. 

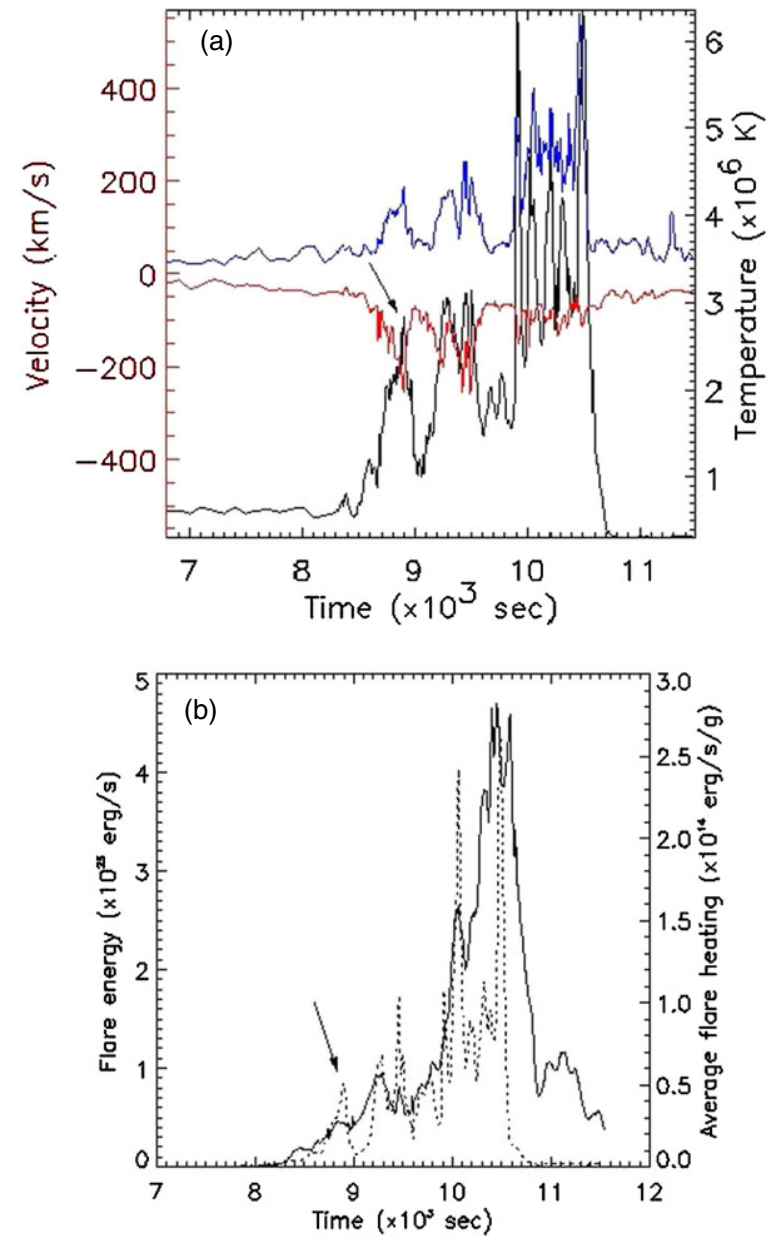

Figure 5. (a) Temporal evolution of maximum temperature and maximum (blue)/minimum (red) vertical velocity at the volume interface that surrounds the small flares: $x=[8.6,12.9] \mathrm{Mm}, y=[2.4,7.1] \mathrm{Mm}, z=[2,10] \mathrm{Mm}$. (b) The same for the total flare energy per second (solid line) and the average flare energy per unit mass (dashed line). The arrow indicates the explosive reconnection event at $t=8900 \mathrm{~s}$.

(A color version of this figure is available in the online journal.)

Some of the larger spikes in Figure 5 (b) (e.g., $t \approx 10,050 \mathrm{~s}$, $t \approx 10,500 \mathrm{~s}$, etc., solid line) represent individual energy emissions of $O\left(10^{27}\right) \mathrm{erg}$. These events might account for microflares. However, many of the events with noticeable total energy release (e.g., around $t \approx 8900 \mathrm{~s}, 9300 \mathrm{~s}, 10,400 \mathrm{~s}$ ) is the result of the superposition of small flares, each involving $10^{25}-10^{26} \mathrm{erg}$, which is the nano/microflare energy regime.

For the small flares that occur in the corona, we estimate that the average energy flux in the area of integration (i.e., $4.3 \times 4.7 \mathrm{Mm}$, as in Figure 5) is at least $O\left(10^{6}\right) \mathrm{erg} \mathrm{s}^{-1} \mathrm{~cm}^{-2}$. This estimate, together with the high occurrence rate of flares in the same area, indicates that nano/microflares can provide a non-negligible contribution of heating in emerging flux regions and in the active $\mathrm{X}$-ray corona. Moreover, we estimate that the fast upward propagation of plasma, which originates mainly from the flare regimes, carries a vast amount of Poynting flux into the corona (in the range $1-60 \mathrm{~kW} \mathrm{~m}^{-2}$ over the whole domain, during $t \approx 8400-10,800 \mathrm{~s}$ ), part of which could contribute to the mass loading and driving of the solar wind. We anticipate that the mechanism presented in our numerical experiments may constitute a generic process, which powers eruptive flaring activity of magnetic fields in astrophysical and laboratory plasmas.

This research was supported by the Research Council of Norway through the grant "Solar Atmospheric Modelling" and through grants of computing time from the Programme for Supercomputing, by the European Research Council under the European Union's Seventh Framework Programme (FP7/20072013)/ERC Grant agreement No. 291058 and by computing project s1061 from the High End Computing Division of NASA. The authors acknowledge support by the EU (IEF-272549 grant) and the Royal Society. The authors are also grateful for indepth discussions during the ISSI workshops: "Magnetic flux emergence in the solar atmosphere" (Bern, 2011 February) and "Understanding solar jets" (Bern, 2013 March).

\section{REFERENCES}

Archontis, V., Galsgaard, K., Moreno-Insertis, F., \& Hood, A. W. 2006, ApJL, 645, L161

Archontis, V., \& Hood, A. W. 2013, ApJL, 769, L21

Archontis, V., Hood, A. W., \& Brady, C. 2007, A\&A, 466, 367

Archontis, V., Moreno-Insertis, F., Galsgaard, K., Hood, A. W., \& O'Shea, E. 2004, A\&A, 426, 1047

Asai, A., Yokoyama, T., Shimojo, M., \& Shibata, K. 2004, ApJL, 605, L77

Carlsson, M., \& Leenaarts, J. 2012, A\&A, 539, A39

Chae, J., Qiu, J., Wang, H., \& Goode, P. R. 1999, ApJL, 513, L75

Gudiksen, B. V., Carlsson, M., Hansteen, V. H., et al. 2011, A\&A, 531, A154

Hanaoka, Y. 1996, SoPh, 165, 275

Hannah, I. G., Christe, S., Krucker, S., et al. 2008, ApJ, 677, 704

Howard, R., \& Stenflo, J. O. 1972, SoPh, 22, 402

Hudson, H. S. 1991, SoPh, 133, 357

Isobe, H., Miyagoshi, T., Shibata, K., \& Yokoyama, T. 2005, Natur, 434, 478

Kopp, R. A., \& Pneuman, G. W. 1976, SoPh, 50, 85

Lin, R. P., Schwartz, R. A., Kane, S. R., Pelling, R. M., \& Hurley, K. C. 1984, ApJ, 283, 421

Machado, M. E., Moore, R. L., Hernandez, A. M., et al. 1988, ApJ, 326, 425

Masuda, S., Kosugi, T., Hara, H., Tsuneta, S., \& Ogawara, Y. 1994, Natur, 371,495

McKenzie, D. E., \& Savage, S. L. 2009, ApJ, 697, 1569

Nishizuka, N., Takasaki, H., Asai, A., \& Shibata, K. 2010, ApJ, 711, 1062

Parker, E. N. 1988, ApJ, 330, 474

Porter, J. G., Moore, R. L., Reichmann, E. J., Engvold, O., \& Harvey, K. L. 1987, ApJ, 323, 380

Priest, E. R., \& Forbes, T. G. 2002, A\&ARv, 10, 313

Shibata, K., \& Magara, T. 2011, LRSP, 8, 6

Shibata, K., Masuda, S., Shimojo, M., et al. 1995, ApJL, 451, L83

Shibata, K., \& Tanuma, S. 2001, EP\&S, 53, 473

Shimojo, M., Narukage, N., kano, R., et al. 2007, PASJ, 59, 745

Sykora, J., Hansteen, V., \& Carlsson, M. 2008, ApJ, 679, 871

Sweet, P. A. 1969, ARA\&A, 7, 149

Tsuneta, S. 1996, ApJ, 456, 840

Tsuneta, S. 1997, ApJ, 483, 507

Yokoyama, T., \& Shibata, K. 1995, Natur, 375, 42 